\title{
Modding Tabletop Games for Education
}

\author{
Daisy Abbott ${ }^{[0000-0002-6870-8481]}$ \\ The Glasgow School of Art, Glasgow, UK \\ d.abbott@gsa.ac.uk
}

\begin{abstract}
This paper describes a learning-objective-centric workflow for modifying ('modding') existing tabletop games for educational purposes. The workflow combines existing research for serious games design with novel systematic analysis techniques for learning and game mechanics and gameplay loops to improve the understanding and rigour of the process. A detailed worked example applies the workflow to the development of a serious tabletop game with the educational goal of increasing knowledge and confidence of performing postgraduate literature reviews. Systematic application of the workflow to a real example supports the value of this approach and provides a useful template for educators to follow for increasing the quality and feasibility of self-designed serious games.
\end{abstract}

Keywords: Serious Games, Board Games, Modding, Game-Based Learning

\section{Introduction}

It is now well evidenced that games can be engaging and effective tools for education but that the design of an intervention has as large an effect on success as the medium [1] and that designing effective game-based learning solutions requires significant expertise in both game-design and pedagogy [2]. Educational games which do not successfully combine game design with learning design are ineffective in terms of engagement, learning, or both (often known as a 'spinach sundae': a product which is neither appealing nor good for you [3]). Furthermore, research into game-enhanced learning demonstrates that the different characteristics of different games have discernible effects on the learning behaviours of players [4] and consequently on how well the game achieves its educational purpose [1, 5]. In short, educational game design is complex, resource intensive, and requires multiple interdisciplinary skillsets. Games designed for digital platforms also need significant technical expertise and the resources to support them. Despite these barriers, the well-documented advantages of game-based learning (GBL) drive demand for games for learning, training, or behavioural change (henceforth referred to as 'serious games') across a wide range of contexts [6-8]. However, as evidence of the efficacy of GBL grows, the discipline gains new advocates from a variety of backgrounds which increases the risk of GBL solutions being designed and implemented in isolation of the expertise and resource contexts necessary to make them effective. This paper presents a practical solution to the tension between the growing desire for serious games amongst students and educators and the barriers to effectively implementing them [9]. It is proposed that serious game design can be streamlined and 
made cost-effective, without the loss of either learning objectives or game engagement and enjoyment, by developing educators' skill in modifying existing tabletop games.

\subsection{Why tabletop games?}

Tabletop games (e.g. board, card, and dice games) are under-represented in serious games literature; in fact, many definitions inexplicably restrict the concept to digital games. For clarity, this paper defines 'games' according to Juul's six game features (games have rules; variable, measurable outcomes with different values; players invest effort and are attached to outcomes; and consequences are negotiable) [10] and 'serious games' as games which have at least one characterizing goal as well as entertainment, regardless of platform.

Clearly, delivery platform (like game mechanics) affects interaction and therefore learning behaviours. Major advantages of digital games are their infinite reproducibility, scalability, and remote digital accessibility. However, if this is not needed - as is the case in some classroom delivery - the interaction behaviours of tabletop games can make them much more appropriate to a wide range of teaching situations. Tabletop interaction is kinaesthetic as well as mental, often involving players literally constructing maps, hands of cards, structures, or patterns on the game board where options are explored and solutions reinforced by physical movement and positioning. Unlike the typically isolated, human-computer-interaction gameplay of educational video games, tabletop games are usually social experiences where players analyse, learn from, and react to the strategies and actions of others. Furthermore, the educator is not only present but an active facilitator when students play educational tabletop games - allowing a more scaffolded learning experience that can be adapted on-the-fly to players' needs and also encourages further learning activities to take place in and around the game context. Enhanced scaffolding has been shown to have a significant improvement on acquisition of intended learning outcomes (ILOs) [1] and instructional support during gameplay is recommended [11]. These characteristics of tabletop games can increase the well-documented educational advantages of digital serious games, particularly for understanding complex systems [12] and collaborative group approaches [1] in tutorials or closely guided classroom contexts.

Furthermore, a common misconception that digital games are inherently engaging and motivating for learners is not supported by evidence. Even well-designed digital serious games can be disengaging, even intimidating, for some learners [7, 13] (particularly in Higher Education [14]) and there is evidence of lack of confidence for teachers using digital serious games, as learning to facilitate the game requires non-trivial additional expertise [13, 15]. This barrier is reduced (albeit not eliminated) for tabletop games, partly due to their lack of technological interface but also because rules and components are explicit and transparent [12]. Technical barriers to engagement should not be underestimated in educational settings. Not only do computer games require hardware, software, power, and often network connections to run at all (infrastructure that is often assumed but rarely smooth for educators to implement [13]), many digital serious games struggle to satisfy the user expectations created by the commercial game industry [13]. 
Finally, tabletop games require no programming skill and can be relatively quick and cheap to develop. This makes them considerably more achievable for educators who have games experience but limited time and money to devote to designing GBL activities. Nevertheless, for tabletop GBL to be successful, it still requires considerable pedagogical and game design expertise. This is the challenge this paper addresses.

\subsection{Why mod?}

Flexibility and customisability are key features of tabletop games that can increase enjoyment [16] and potentially learning. The conceptual approach here assumes that pedagogy experts have familiarity with games, the desire to create GBL activities for their students, but not necessarily any applied game design expertise. It is concentrated on three principles:

1. Educators could focus on adapting ('modding') existing tabletop games, instead of creating a game from scratch. This approach reduces the game design expertise required (modding skill relies on recognizing/adapting rather than conceiving/designing appropriate game mechanics) and eliminates the need for lengthy testing, as the game being adapted is already commercially successful. Of course, adaptations for learning will still require evaluation and it is important to acknowledge that game literacy is important as misrecognition of game mechanics may impair learning. However, modding markedly reduces the expertise and resources required to produce an effective serious game.

2. Serious game design is made more manageable by adapting previous processes and workflows, concentrating on modding. This provides robust design principles that are easy to understand and follow, whilst also giving educators the framework for the baseline skills required for serious game design and a context for improvement.

3. The incorporation of analysis techniques that can be relatively easily understood and applied allows pedagogy experts to swiftly gain the minimum expertise necessary to produce and implement a serious game without too onerous a demand on their time.

The remainder of this paper presents a workflow for serious game modding, aimed at educators with some experience of GBL, which is critically applied via a worked example (identified as important for understanding $[9,15])$. The game developed elucidates the process for a particular, real-world educational setting; increasing knowledge and confidence for Higher Education students about to undertake a literature review.

\section{Workflow for serious game modding}

Fig. 1 shows a workflow for effectively modding tabletop games for educational contexts. This workflow synthesises previous serious game development models [4, 5, 1719], incorporating guidelines aimed at educators [13, 20], with a particular focus on tabletop GBL [6] and modding [12]. It further enhances previous research by categorising the steps by pedagogic and/or game design expertise and including gameplay 
loop analysis [21] as a method to map learning mechanics to game mechanics [22] in order to inform both choice and adaptation of games. Each step is described below.

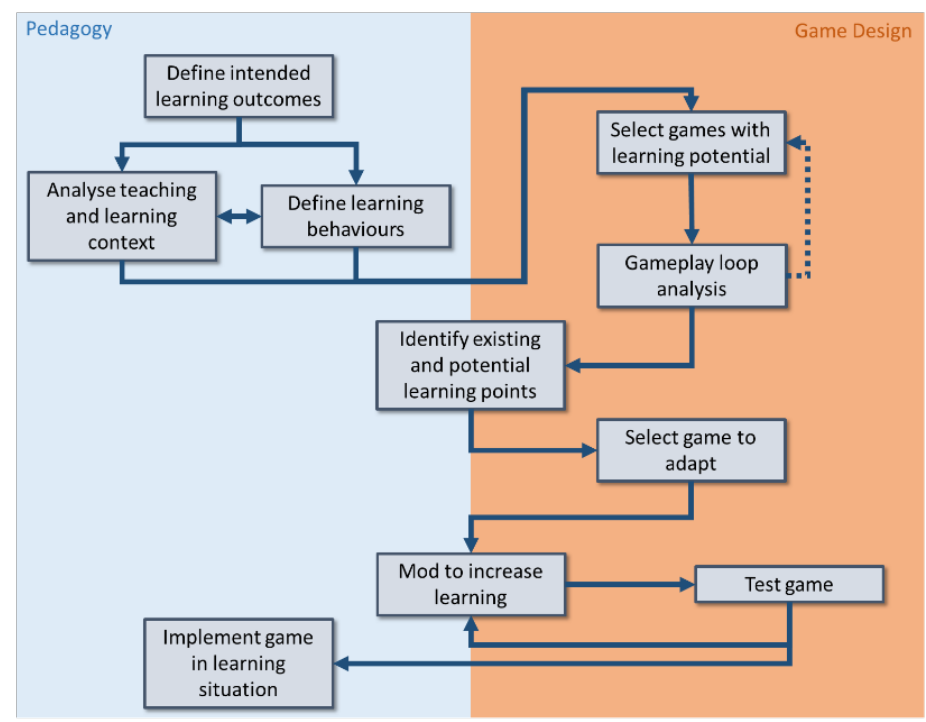

Fig. 1. - Workflow for modding existing games for educational purposes

\section{Define learning outcomes}

The first (and most important) step is to define the overall purpose of the activity in terms of its intended learning outcomes (ILOs): i.e. what knowledge or understanding should be improved after playing? This may seem obvious but is worth making explicit that ILOs should be clearly defined and kept in mind throughout the design process. If this step is taken for granted, or subsumed into game design, it is likely that the game will either not be effective, or will teach something other than the lessons intended. This primary and central position of ILOs reflects previous research cited above.

\section{Analyse context and define learning behaviours}

The next, concurrent, steps are to consider the learning and teaching context and to define the intended learning behaviours [4, 17, 23]. Too often games are developed in isolation of their context and not enough thought is given to how they will be effectively integrated into teaching and learning practice $[13,17]$. Is the game intended for quiet, independent study or will it be deployed in a classroom of 30 children? Will it be played once or repeated? Will players compete or co-operate? Who will lead the game and support the learning? A common mistake in designing serious games is to default to a 'question and answer' model, familiar to educators from quizzes and simple trivia games. This is perfect for demonstrating existing knowledge but ineffective for learning new information [5]. Therefore, if ILOs are centred around memorisation, the learning mode is behaviourist 'drill and practice', and the context is independent home study, a digital game with strong extrinsic rewards, high interest and replayability, or both, 
could be an effective solution [11, 24]. Conversely, if ILOs rely on collaborative, constructivist learning behaviours and the context is a one-hour tutorial for postgraduates, this will require very different gameplay to achieve best results [23]. Understanding how as well as what the students are expected to learn is crucial to informing all the game design steps that follow. A useful guide for GBL novices can be found at [20].

\section{Select potential games}

Next, create a shortlist of games that have potential for delivering the ILOs defined, in the way that fits the learning situation. For educators with limited experience of games, this can be daunting, however it arises directly from the analysis of the learning behaviours and situation and becomes easier as modders increase their game literacy [12]. Broadly speaking, modders should consider the overall metaphor for the game as indicated by the learning behaviours, for example, collaborative problem-solving, competitive race, resource management, creative interpretation, or map-building. To then shortlist suitable games it is useful to use published classification systems such as the Gameplay / Purpose / Scope model [25], fan-produced tabletop game taxonomies ${ }^{1}$ and (not to be overlooked) one's own experience and recommendations. It is also recommended for educators to involve their students in the design stage, as much as is possible, not only for acceptance but students themselves may have valuable insights. Importantly, the overall game format should arise from learning behaviours and context, not the subject matter. Whilst it may be tempting to search for games with similar content to the ILOs, it is the underlying game mechanics that support learning and there arises a risk that the game 'skin' (i.e. theme/narrative) will distract from the actual pedagogic goals.

Gameplay loop analysis, identification of learning points, game selection

Gameplay refers to players interacting with the game and here explicitly includes cognition as well as physical actions influencing the game world. The gameplay loop follows Guardiola's definition [21] and builds on his pedagogic method: the loops represent gameplay as linked actions. This process breaks down and describes every game interaction, allowing educators to consider the types of behaviours involved and map them to learning behaviours. This stage is more easily understood using the worked example (Fig. 2), however the basic steps for analysis are as follows. Firstly, play the game. Whilst aspects of gameplay loops can be discerned from reading the rules, it is necessary to gain a more holistic understanding through play as this includes social and emotional elements of gameplay. Next, map the gameplay loops as a flowchart at a macro level, identifying In Game and Out Game actions [21] i.e. actions that have an immediate, measurable effect in the game such as moving a piece, and those that do not such as chatting to other players. As much as is productive, expand each sub-loop and categorise the types of interactions using an established concept map such as the Game Mechanic - Learning Mechanic framework [22]. This allows the identification of learning mechanics that happen in and between each interaction (not forgetting Out Game

\footnotetext{
${ }^{1}$ E.g. https://boardgamegeek.com/thread/581158/alternative-classification-board-games-long; https://www.boardgamegeek.com/image/3613899/rouie-a
} 
actions, where important scaffolding and metacognition takes place). Finally, highlight points that would easily support additional learning mechanics, even when not currently present in gameplay. Based on these analyses, it is possible to see which games have the mechanics that best support the learning behaviours desired (or, to demonstrate that games do not support the overall purpose, in which case it may be necessary to return to the shortlisting stage or elect instead to create the game from scratch).

\section{Modification for learning}

If analysis shows that all learning mechanics are already present and the game's theme can be mapped directly to the stated ILOs, modifying a game could be as simple as changing the theme content ('reskinning'). However, it is more likely that game mechanics will need to be added to or altered to enable or reinforce the desired learning mechanics and players' exposure to learning material (content) during gameplay. A simple example is creating a trivia game by adding 'question and answer' interaction which acts as a gateway to progression. Whilst this 'quiz' approach can be very effective for certain ILOs, it has limitations [5] whereas gameplay with intrinsically integrated learning content can be more effective for learning and motivation [26]. Therefore care should be taken to, where fruitful, embed learning mechanics into the core of gameplay without disrupting the overall game. In general, once gameplay satisfies the ILOs, context and behaviours defined, game mechanics should be altered as little as possible as every change affects the game system and balance and could lead to unwanted emergent behaviours or even risk undermining the ILOs.

\section{Testing and implementation}

The modded game should be iteratively tested and improved to ensure firstly that the modifications have not 'broken' the game and, crucially, that the game does impart the ILOs stated. Then the game can be implemented in context.

\section{Worked example: a game to teach literature review skills}

The serious game in this example arose directly from a practical problem: students on a postgraduate Research Skills course are required to learn how to perform a literature review but many lacked confidence and knowledge of how to begin and felt overwhelmed by the scale of this complex process, which impaired their learning. This pedagogic 'vertigo' can be very fruitfully mirrored and addressed by tabletop games. Whilst it is usually recommended to minimise learning about the game in order to maximise learning through the game [27], games systems can model larger complex systems and "when the sim dissolves the player's game vertigo, it also dissolves his system vertigo about the large and complex system that the sim is modelling." [12] Therefore, a GBL approach was chosen to reflect the process of undertaking a literature review and to diffuse students' trepidation about starting this large and complex task.

Intended learning outcomes 
Intended learning (and attitudinal) outcomes were defined as follows. After playing the game, students should: Understand the basic principles of searching, evaluating, and assimilating research sources; Be able to identify different types of source and understand when they are most useful; Be able to describe techniques for choosing sources and deciding what to read; Recognise that evaluation of sources has a time cost, but saves time and improves quality overall; Understand that literature search and review requires iterative refocussing and describe techniques for refining searches, and; Feel more relaxed and confident about the literature search and review process.

\section{Teaching and learning context}

Implementation is within a postgraduate tutorial context, therefore a face-to-face, instructor-supported tabletop game is suitable. Student numbers exceed 30 therefore the game should either be simple enough to not require direct supervision or engaging enough to maintain interest and learning as a spectator. An alternative is to implement the game with smaller groups in turn whilst the rest of the class are engaged in a different activity, requiring the game to be relatively short duration. Supporting smaller groups of players is an acknowledged limitation of non-digital tabletop games with implications for educators that must be considered.

\section{Learning behaviours}

Learning behaviours were defined to mirror the real-life task of performing a literature review: exploratory, discovering, constructivist, independent, selective, refining/refocussing, consolidating, and non-linear. Overall comprehension of the process is more important than learning specific facts, therefore repetition is focussed on refining understanding, not memorisation. Due to students feeling overwhelmed, it is also important to 'drip feed' content, using a Cascading Information learning mechanic [22].

\section{Shortlist games}

Games were shortlisted based on a strategic construction overall mechanic (as a metaphor for exploring and constructing an understanding of a research topic through a literature review), including elements of selection and matching (representing selection of sources to match research topic), and repetition for both consolidation of knowledge/understanding and refining. Five games were shortlisted using BoardGameGeek’s taxonomy and personal recommendations: Blueprints, Meeple Circus, Best Treehouse Ever, Junk Art, and Rhino Hero. ${ }^{2}$ All five games involve literally constructing something and for four games, Dexterity is a core mechanic meaning game tokens are played thoughtfully and carefully. All are competitive (with the exception of one of the minigames within Junk Art), however in all cases except Rhino Hero, competition

\footnotetext{
${ }^{2}$ https://www.zmangames.com/en/products/blueprints/; http://www.matagot.com/en/catalog/details/family-games/3/meeple-circus/894; http://www.greencouchgames.com/besttreehouseever; http://www.pretzelgames.com/en/home/21-junk-art.html; http://www.habausa.com/rhino-hero/
} 
is via scoring each player's own structure rather than directly interfering with opponents' structures. All games except Rhino Hero explicitly involve repetition and Cascading Information is core to Meeple Circus and Best Treehouse Ever.

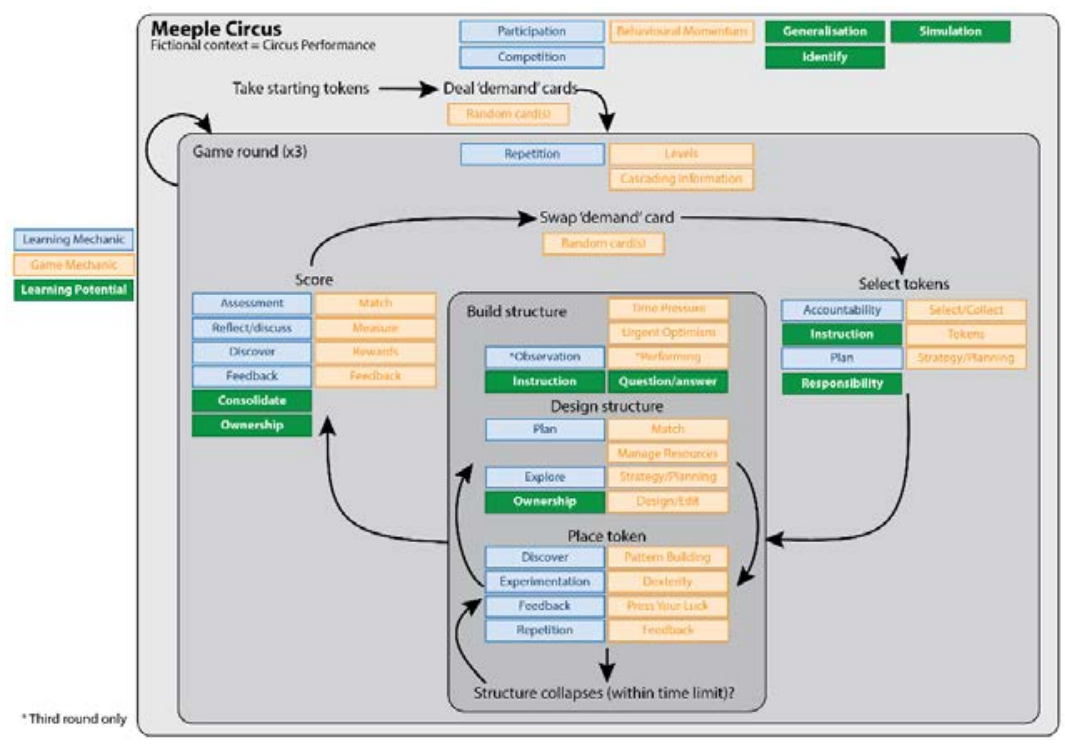

Fig. 2. - gameplay loop analysis of Meeple Circus, using an extension of the LM-GM model

Gameplay loop analysis and selection

Guided by the literature, gameplay loops were produced for each shortlisted game (including every minigame within Junk Art) and points of learning (and potential learning) were identified. This allowed a rigorous parity of comparison between each game.

Rhino Hero was rejected first as the lose condition is binary - when the tower collapses, the game ends for everybody - this halts learning. Analysis showed that this game relies heavily on Dexterity and Press Your Luck mechanics which were difficult to imagine being intrinsically integrated with the ILOs, and learning mechanics are restricted to planning which cards to use against your opponents. However, its simplicity, linearity, and quick repeatability would be highly appropriate for lower-order ILOs related to literature review, especially as the folded cards are reminiscent of books.

Junk Art was also rejected. Whilst several of the minigames were highly appropriate for the fictional context of performing a literature review, token design made balancing pieces more difficult which, considered alongside the core role of fallen pieces in most of the minigames' exit conditions and scoring, was felt to not contribute to the learning behaviours effectively. A further reason was that scoring rewards and penalties are explicitly comparative (e.g. who has the tallest sculpture and/or most fallen tokens), whereas the scoring of the remaining three games focusses more on how well each individual construction matches external scoring conditions. The latter situation was judged to be more appropriate to the literature review metaphor. 
Blueprints, Meeple Circus, and Best Treehouse Ever were all suitable for modding for the ILOs and context defined. All three use repetition, strategic selection and management of resources, strategic placement of tokens/tiles in the overall construction, followed by a scoring phase which allows assessment, feedback, and reflection for players. Furthermore, all three involve design for pattern matching with strategic elements. Meeple Circus uses an elegant Cascading Information mechanic via game Levels to gently introduce complexity, thereby reducing game vertigo whilst also acting as a highly appropriate metaphor for the iterative refining process of exploring a research topic. Furthermore, overall its rules were felt to be simpler than the other two games, therefore Meeple Circus (Fig. 2) was selected as the game to be modded.

\section{Mod game to increase learning}

The first step was to change the theme to match the learning situation. Instead of creating a circus performance, players are building their knowledge of a research area by finding, reading, and synthesising literature and other research sources. Strategic selection of game tokens becomes searching and evaluating sources, placing tokens in the structure represents understanding and synthesising knowledge, matching acrobatic feats becomes closeness of fit to the research question being investigated, and scoring indicates the quality of the literature review. In this way, the game functions as a simulation, allowing players to both identify with the content and generalise it to their individual situations (Fig. 2).

Care was taken when conceptually 'reskinning' the game to map components to ILOs which are supported by the game rules, particularly the scoring system. For example, a blue acrobat represents baseline knowledge sources used when starting a literature search - because it scores points for touching the ground it is much more useful in the first round than a red acrobat, which represents innovation and originality and scores highly in later rounds. 'Audience demand' cards score when a player matches their pattern - this represents the student's current use of sources to understand their research area - and changes between rounds, making it an excellent metaphor for iteratively developing an understanding of a specific topic. The change in game theme requires explanation to students, therefore an Instruction learning mechanic is required in the Select and Build phases.

The next step was to evaluate how well the reskinned game matches the ILOs. Each ILO was mapped to existing game mechanics to assess whether it was delivered. Where not effectively achieved, additional learning and/or game mechanics were devised using the points of potential learning already identified in the gameplay loop analysis. Additional game mechanics were required to deliver the Instruction, Question/Answer and Consolidate learning mechanic potential. To ensure all ILOs were satisfied, the following rules were added:

1. When taking any component, players must first draw the appropriate card for that component from the new deck of Instruction cards and read out the fact/tip to the group. Cards contain ideas for sources of this particular type, and techniques for evaluating sources and deciding what (and what not) to read. This builds knowledge 
which can be later demonstrated or discussed in 'out game' learning activities with direct relevance to the student's particular subject domain.

2. After component selection, players have the option of receiving a generic extra component (such as a 6-sided die) or of swapping one of their existing components for one of their choice (after considering a question from a new deck of Evaluate cards). This mechanic creates active engagement with the techniques of rigorous evaluation of sources. However, this mechanic in particular needed to be tested to ensure that swapping leads to higher scores than accepting the generic component.

3. During the 'swap demand card' phase, the two (as opposed to one) players with the lowest score can swap an 'audience demand' card. To do this each must read out a card from the new deck of Refine and Focus cards. This increases emphasis on techniques for refining and focussing research questions.

4. The final round contains performative 'challenge' cards. These cards were altered to allow players to demonstrate knowledge about specific aspects of the literature review process, as well as learning from each other. They move cognitive processes explicitly into the active mode by prompting recall, analysis, or interpretation of game content.

5. Finally, the name of the game was changed to On the Shoulders of Giants.

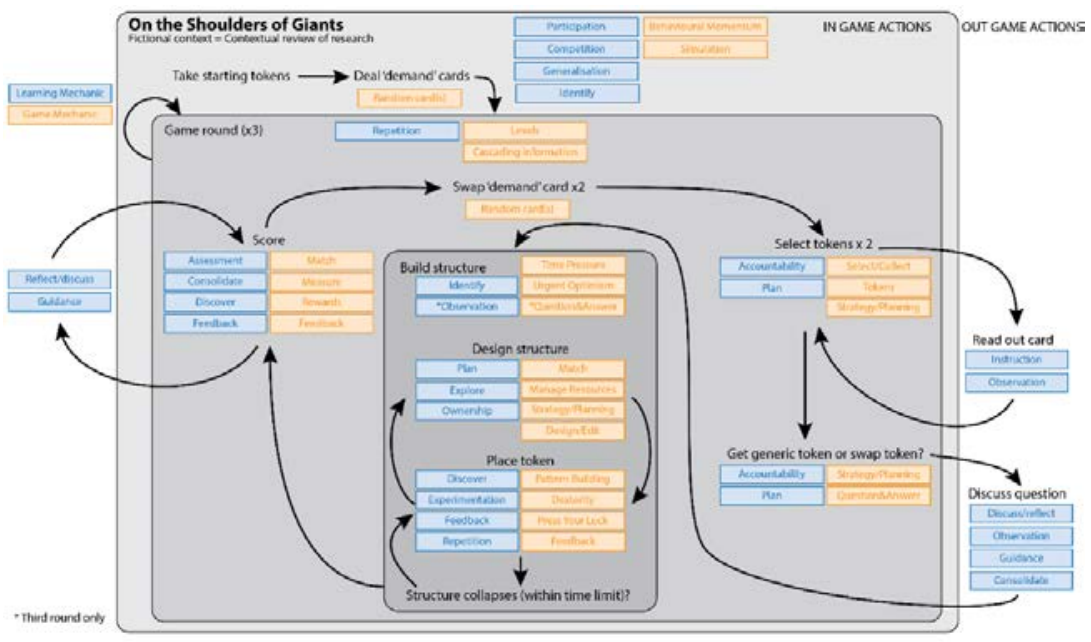

Fig. 3. Gameplay loop analysis of On the Shoulders of Giants: educational mod of Meeple Circus

On the Shoulders of Giants was then informally tested with small numbers of students and staff who teach research skills and feedback on gameplay and the content and phrasing of learning cards was incorporated. No major changes were required.

\section{Conclusion and next steps}

This worked example of the development of a tabletop serious game shows the value of using a robust design workflow to modify an existing tabletop game. Reflection on 
the process demonstrates that close analysis using the LM-GM model, combined with gameplay loops, allows the identification of particular points within gameplay where educational content can be integrated to match the game's content to defined ILOs. This makes the process of game design significantly easier and more rigorous, especially for modders who do not necessarily already have a high level of game literacy.

Further steps are to formally evaluate this game with a larger cohort of students in order to demonstrate whether or not it is effective at delivering the ILOs and attitude change defined. Another avenue for future research would be to test this workflow with educators who have limited experience of game design, to establish if their experience supports the hypothesis that serious game design can be achievable (albeit still challenging) by analysing and modding tabletop games in a systematic and rigorous way.

Finally, incorporating feedback from the serious games research community has the potential to further improve and potentially streamline this workflow to increase its efficacy and ease of use.

Game rules and further information about this research is available at http://blog.gsofasimvis.com/index.php/research/games/

\section{References}

1. Clark DB, Tanner-Smith EE, Killingsworth SS (2016) Digital Games, Design, and Learning: A Systematic Review and Meta-Analysis. Review of Educational Research 86:79-122 . doi: 10.3102/0034654315582065

2. Bellotti F, Kapralos B, Lee K, et al (2013) Assessment in and of Serious Games:An Overview. In Advances in Human-Computer Interaction Volume 2013 2013:1-11 . doi: $10.1155 / 2013 / 136864$

3. Jenkins H, Squire K, Tan P (2004) "You can’t bring that game to school!” Designing supercharged! In: Laurel B (ed) Design research: Methods and perspectives. MIT Press, Cambridge, MA, pp 244-252

4. Grey, Simon and Grey D and, Gordon, Neil and Purdy J (2017) Using Formal Game Design Methods to Embed Learning Outcomes into Game Mechanics and Avoid Emergent Behaviour. International Journal of Game-Based Learning 7:63-73

5. Nicholson S (2011) Making the Gameplay Matter: Designing Modern Educational Tabletop Games. Knowledge Quest 40:60-65

6. Sardone NB, Devlin-Scherer R (2016) Let the (Board) Games Begin: Creative Ways to Enhance Teaching and Learning. The Clearing House: A Journal of Educational Strategies, Issues and Ideas 89:215-222 . doi: 10.1080/00098655.2016.1214473

7. Bourgonjon J, Valcke M, Soetaert R, Schellens T (2010) Students' perceptions about the use of video games in the classroom. Computers and Education 54:1145-1156 . doi: 10.1016/j.compedu.2009.10.022

8. Sandford R, Ulicsak M, Facer K, Rudd T (2006) Teaching with Games. Bristol

9. Ney M, Emin V, Earp J (2012) Paving the way to game based learning: A question matrix for teacher reflection. Procedia Computer Science 15:17-24 . doi: 10.1016/j.procs.2012.10.053

10. Juul J (2003) The game, the player, the world: Looking for a heart of gameness. In: Copier 
M, Raessens J (eds) Proceedings of the Level Up: Digital Games Research Conference. University of Utrecht, Utrecht, pp 30-45

11. Hays RT (2005) The effectiveness of instructional games: a literature review and discussion. Naval Air Warfare Center Training Systems Division 1-63 . doi: citeulike-article-id:3089090

12. Castronova E, Knowles I (2015) Modding board games into serious games: The case of Climate Policy. International Journal of Serious Games 2:41-62 . doi: dx.doi.org/10.17083/ijsg.v2i3.77

13. Marklund BB (2014) Working with Educational Games. University of Skövde

14. Whitton N (2011) Encouraging Engagement in Game-Based Learning. International Journal of Game-Based Learning 1(1):75-84 . doi: 10.4018/ijgbl.2011010106

15. Bourgonjon J, De Grove F, De Smet C, et al (2013) Acceptance of game-based learning by secondary school teachers. Computers and Education 67:21-35 . doi: 10.1016/j.compedu.2013.02.010

16. Frapolli F, Malatras A, Hirsbrunner B (2010) Exploiting traditional gameplay characteristics to enhance digital board games. In: 2nd International IEEE Consumer Electronic Society Games Innovation Conference, ICE-GIC 2010. IEEE, Hong Kong

17. Catalano CE, Luccini AM, Mortara M (2014) Best Practices for an Effective Design and Evaluation of Serious Games. International Journal of Serious Games 1: . doi: 10.17083/ijsg.v1i1.8

18. Marne B, Wisdom J, Huynh-Kim-Bang B, Labat J-M (2012) The Six Facets of Serious Game Design: A Methodology Enhanced by Our Design Pattern Library. In: Proceedings of the European Conference on Technology Enhanced Learning. pp 208-221

19. Lui RWC, Au CH (2018) Establishing an Educational Game Development Model. International Journal of Game-Based Learning 8:52-73 . doi: 10.4018/IJGBL.2018010104

20. Torrente J, Marchiori EJ, Blanco A Del, et al (2011) Production of Creative Game-Based Learning Scenarios: A Handbook for Teachers

21. Guardiola E (2016) The Gameplay Loop: A Player Activity Model for Game Design and Analysis. In: Proceedings of the 13th International Conference on Advances in Computer Entertainment Technology. Osaka

22. Lim T, Louchart S, Suttie N, et al (2013) Strategies for Effective Digital Games Development and Implementation. In: Baek Y, Whitton N (eds) Cases on Digital Game-Based Learning: Methods, Models, and Strategies. IGI Global, Hershey, PA, pp 168-198

23. Abbott D (2015) "How to fail your research degree a serious game for research students in higher education"

24. Keller JM (2009) Motivational Design for Learning and Performance: The ARCS Model Approach. Springer, New York Dordrecht Heidelberg London

25. Djaouti D, Alvarez J, Jessel J-P (2011) Classifying serious games: The G/P/S model. In: Patrick Felicia (ed) Handbook of research on improving learning and motivation through educational games: Multidisciplinary approaches. IGI Global, Hershey, PA, pp 118-136

26. Habgood MPJ, Ainsworth SE (2017) Motivating Children to Learn Effectively : Exploring the Value of Intrinsic Integration in Educational Games Motivating Children to Learn Effectively : Exploring the Value of Intrinsic Integration in Educational Games. 8406: . doi: 10.1080/10508406.2010.508029

27. Sandford R, Williamson B (2005) Games and learning/A handbook from Futurelab. Bristol 